\title{
Aspectos de Personalidade em Adolescentes Infratores Violentos e Não Violentos segundo Inventário de Jesness: Um Estudo Comparativo
}

\section{Personality Aspects in Violent and Nonviolent Juvenile Offenders According to Jesness Inventory: A Comparative Study}

\author{
Ana Luiza Mendonça dos Santos (orcid.org/0000-0002-0736-5024) ${ }^{1}$ \\ André Vilela Komatsu (orcid.org/0000-0001-8508-6787)2 \\ Marina Rezende Bazon (orcid.org/0000-0002-8037-8710)³
}

\begin{abstract}
Resumo
Pesquisas criminológicas destacam as variáveis relativas a aspectos da personalidade como os melhores preditores do comportamento delituoso persistente. Este estudo objetivou verificar se adolescentes infratores violentos e não violentos apresentam padrões de personalidade diferentes, avaliados pela versão brasileira do Inventário de Jesness. Cento e vinte e nove adolescentes do sexo masculino em conflito com a lei, de 15 a 18 anos, com histórico de três boletins de ocorrência, em média, foram divididos entre os que haviam cometido delitos violentos ( $G V=88)$ e os que não haviam $(G N V=41)$. Os grupos foram comparados nas 13 escalas do Inventário e os resultados indicaram diferenças significativas entre GV e GNV em seis das 13 escalas. Do ponto de vista da interpretação do instrumento e suas escalas, GV pontuou em um sentido pior que GNV. As escalas que diferenciaram os grupos relacionam-se a um funcionamento psicológico que remete a problemas de controle dos impulsos, manejo da raiva/sentimentos negativos e atitudes hostis/agressivas.
\end{abstract}

Palavras-chave: Adolescentes em conflito com a lei. Violência. Personalidade. Inventário de Jesness.

\begin{abstract}
Criminological research highlights variables related to personality aspects as the best predictors of persistent criminal behavior. This study aimed to verify if violent and non-violent adolescent offenders show different personality patterns, evaluated by the Brazilian version of the Jesness Inventory. 129 male adolescents in conflict with the law, aged 15 to 18 years, with a history of 3 offense records on average, were divided between those who had committed violent crimes (GV = $88)$ and those who had not $(\mathrm{GNV}=41)$. The groups were compared in the 13 scales of the Inventory. The results indicated significant differences between GV and GNV in 6 of the 13 scales. Regarding the interpretation of the instrument and its scales, GV scored in a worse direction than GNV. The scales that differentiate the groups are related to psychological
\end{abstract}

\footnotetext{
11 Universidade de São Paulo, São Paulo, Brasil. E-mail: almsantos@outlook.com.br.

22 Universidade de São Paulo, São Paulo, Brasil. E-mail: avk@usp.br.

33 Universidade de São Paulo, São Paulo, Brasil. E-mail: mbazon@ffflrp.usp.br. 
functioning that refers to problems of impulse control, anger/negative feelings management and hostile/aggressive attitudes.

Keywords: Juvenile delinquency. Violence. Personality. Jesness Inventory. 


\section{Introdução}

O conceito de violência pode ser bastante amplo, sendo utilizado cotidianamente para descrever um grande leque de situações, mas a Organização Mundial da Saúde (OMS, 2002, p. 5) a define como "uso intencional de força física ou de poder, real ou em ameaça, contra si próprio, contra outra pessoa, ou contra um grupo ou uma comunidade, que resulte ou tenha possibilidade de resultar em lesão, morte, dano psicológico, deficiência de desenvolvimento ou privação". Essa definição refere-se, portanto, a situações de violência interpessoal, na qual o comportamento do autor impacta (ou tem possibilidade de impactar) direta e quase que imediatamente na saúde da vítima. Essa modalidade de violência está entre os problemas sociais que mais preocupam os brasileiros. Segundo o Atlas da Violência (Cerqueira et al., 2017), mais de 59 mil pessoas morrem anualmente vítimas de homicídio no país. Essa alta incidência provoca mudanças de hábitos que certamente reduzem a qualidade de vida das pessoas, conforme aponta a Pesquisa Nacional de Vitimização (Datafolha, 2013), na qual 91\% dos entrevistados relataram já ter deixado de ir a algum lugar ou evitado alguma situação por medo da violência. Assim, apresenta-se essencial a tarefa de identificar e entender os fatores que facilitam ou promovam a manifestação de comportamentos violentos para que medidas protetivas sejam tomadas, rumo a um convívio social e relacional mais harmonioso.

Nesse cenário em que comportamentos violentos causam prejuízos para a saúde e bem-estar individual e coletivo, os jovens desempenham um importante papel que pode ser analisado sob duas perspectivas distintas: como vítimas e/ou como agentes da violência. Como vítimas, os registros de saúde de 2012 apontam que a violência é a principal causa de morte entre pessoas de 15 a 29 anos, responsável por 39\% dos óbitos nessa faixa etária no Brasil (Waiselfisz, 2014, p. 20). Como agentes, os registros criminais mais recentes divulgados pelo Fórum de Segurança Pública (2017), relativos a essa população, revelam que adolescentes foram responsáveis por 3.951 homicídios, tentativas de homicídio ou latrocínio e por 12.196 roubos, tentativas de roubo ou lesão corporal. Acrescentase, ainda, que tais números podem ser ainda maiores, considerando que a autoria de muitos crimes não é identificada.

Sabe-se que condutas antissociais são bastante comuns na adolescência. Estudos em diferentes contextos socioculturais - europeu, africano, norte-americano e, especificamente, brasileiro - evidenciam que uma proporção considerável de jovens, a maioria dos adolescentes do sexo masculino, apresenta comportamentos divergentes (contravenções) e/ou delituosos (infrações penais), o que permite considerar tais comportamentos como parte de um processo de desenvolvimento típico (Castro, Cardoso \& Agra, 2010; Cook, Pflieger, Connell \& Connell, 2015; Dias, Formiga \& Gonçalves, 2016; Komatsu \& Bazon, 2015; Le Blanc, 2003). Em conjunto com atividades regulares para a idade, esses comportamentos permitem ao jovem explorar e se apropriar do ambiente em que está inserido, ajustando-se à condição de relativa independência em relação à família e gradativa autonomia. Esse tipo de envolvimento com atividades 
delituosas/infracionais é chamado na literatura especializada de delinquência comum, a qual remete a um comportamento ocasional nas experiências vividas pelo jovem, não necessitando de intervenções específicas, pois, com o tempo, cessaria naturalmente ainda na própria adolescência (Le Blanc, 2003; Moffitt, 1993).

Contudo, para uma parcela de jovens, o comportamento antissocial, especificamente o delituoso/infracional, seria mais frequente $\mathrm{e}$ diversificado, implicando, por vezes, em um aumento de violência de natureza interpessoal, caracterizando o que a literatura especializada chama de delinquência persistente (ou distintiva) que remete a padrões de comportamentos antissociais, desenvolvidos ao longo do tempo, sustentados pela exposição do adolescente a uma série de fatores de risco contextuais (oportunidades antissociais e vivências estressantes em diferentes contextos de socialização, por exemplo) e sociais (conflitos familiares, histórico de punição escolar e afiliação a pares antissociais, por exemplo), somados à escassez de fatores protetivos (apoio parental e vínculo escolar ou laboral, por exemplo), que interferem negativamente no seu desenvolvimento pessoal, acarretando defasagens em diferentes áreas que limitam o potencial desses jovens (Loeber, Farrington \& Petechuk, 2013; Komatsu \& Bazon, 2018; McGee \& Farrington, 2015).

Nessa linha de raciocínio, entre as diferentes dimensões do desenvolvimento que podem ser afetadas no escopo da delinquência persistente, está a personalidade, em alguns de seus atributos, segundo estudos de revisão e de metaanálise (Andrews \& Bonta, 2010; Gendreau, Little \& Goggin, 1996). Esses atributos remetem a um conjunto de características/traços que influenciam em como o indivíduo interage com o ambiente. $\mathrm{Na}$ esfera da delinquência persistente, esses atributos de personalidade ganham uma orientação antissocial, que favorece um comportamento também antissocial, sendo, portanto, importantes fatores de risco à prática de delitos. Adicionalmente, alguns desses traços de personalidade estão ligados especificamente ao comportamento delituoso violento (Moffitt, Krueger, Caspi \& Fagan, 2000).

Larsen e Buss (2009) definem a personalidade como o conjunto de traços e mecanismos psicológicos de um indivíduo, organizados e relativamente estáveis, que medeiam sua interação e adaptação aos ambientes intrapsíquico, físico e social. Nesse sentido, os traços psicológicos são características individuais e representam a tendência geral de comportamentos de uma pessoa, a depender do contexto em que está inserida. Essa perspectiva baseada no modelo de traços é útil para descrever e entender as diferenças entre as pessoas; explicar comportamentos (como funções dos traços); e predizer comportamentos, na medida em que os padrões de comportamento de cada indivíduo tendem a se repetir em contextos semelhantes. Dessa forma, quando a interação e a adaptação do adolescente ao ambiente - mediadas pelos traços de personalidade - ocorrem de maneira pouco adequada ou conflituosa, estas podem favorecer a utilização de comportamentos violentos perante a diferentes exigências sociais como: (i) relações interpessoais; (ii) resolução de problemas; (iii) busca por satisfação de necessidades, desejos ou interesses de forma rápida ou imediata; e (iv) para alcançar outros objetivos (Manzi-Oliveira, 2012; Komatsu \& Bazon, 2017; Tedeschi \& Felson, 1994). É importante ressaltar que, de uma perspectiva desenvolvimental, os traços são 
modelados por um processo contínuo de interação entre organismo e ambiente, de modo que variáveis de natureza biológica (características físicas, temperamento, estágio desenvolvimental etc.) e de natureza ambiental (alimentação/nutrição, interação familiar, interação escolar, influência cultural etc.) se influenciam mútua e cumulativamente na constituição de padrões relativamente estáveis de perceber, sentir e agir em contextos diversos (Achenbach \& Rescorla, 2006; Andrews \& Bonta, 2010; Larsen \& Buss, 2009; Pickles \& Hill, 2006). Portanto, quando nos referimos à personalidade ou aos traços de personalidade, não o estamos a um constructo inato, imutável ou independente de influências sociais e culturais, mas $\operatorname{sim} a$ características pessoais que se constroem (e se reelaboram) na trajetória de vida de cada indivíduo.

Em relação ao comportamento infracional persistente violento, estudos empíricos e de revisão indicam que os traços de personalidade são de grande relevância para explicar a conduta violenta, em especial traços como afetividade negativa tendência em sentir emoções ruins, como a raiva -, impulsividade e baixo autocontrole - dificuldade em controlar os impulsos que resultam em alta reatividade diante de emoções e tendência em buscar satisfações imediatas em detrimento das de longo prazo -, baixa empatia - pouca capacidade de perceber as emoções e os sentimentos dos outros e hostilidade - traço multidimensional que envolve componentes afetivo (raiva), cognitivo (atitudes) e comportamental (agressão) - conforme Capaldi, Knoble, Shortt e Kim (2012); Díaz-Galván, Ostrosky-Shejet e Romero-Rebollar (2015); Howard (2015); Marshall e Brown (2006); Moffitt, Krueger, Caspi e Fagan (2000); Winter, Spengler, Bermpohl, Singer e Kanske (2017).
Os traços citados são características individuais que denotam uma disposição a agir de determinada maneira, modulando vários aspectos do comportamento e do modo como o cérebro processa informações sociais (Chernyshev, Lazarev \& Chernysheva, 2013). A interação desses traços com certas influências ambientais pode concorrer para o desenvolvimento de uma cognição antissocial orientada à agressividade - padrões de processamento de informações e conjuntos de crenças, valores e atitudes favoráveis à violência em razão da: codificação desajustada de estímulos ambientais; atenção seletiva a gatilhos para uso da agressividade; tendência em atribuir intenções hostis aos outros; interpretação equivocada de diferentes estados de excitação, como estado de raiva; habilidades sociais limitadas para solucionar situações adversas; seleção de soluções dirigidas a ações em detrimento de reflexões; perspectiva egocêntrica; capacidade limitada de perceber o estado emocional do outro; e crenças e valores que apoiam o uso da violência para resolução de problemas e/ou alcançar objetivos (Dodge, 2011; McGuire, 2009; Stoddard, Varela \& Zimmerman, 2015; Winter, Spengler, Bermpohl, Singer \& Kanske, 2017). Esse conjunto de fatores revela estados disposicionais, crenças, valores e atitudes subjacentes às ações do indivíduo. Ou seja, são aspectos de personalidade que denotam uma tendência em comportar-se de forma violenta em determinados contextos.

No campo da avaliação forense, destaca-se o instrumento Inventário de Jesness - Revisado (Jesness, 2003) como um dos mais utilizados na avaliação de adolescentes em relação a traços, crenças, valores e atitudes associados à conduta delituosa (Pinsoneault, 2006). Com base na 
avaliação desses atributos específicos da personalidade, esse instrumento tem se mostrado útil em discriminar adolescentes infratores de adolescentes não infratores (Bazon, 2016; Costa, Komatsu \& Bazon, 2017) e em predizer reincidência em jovens e adultos infratores (Benda, Corwyn \& Toombs, 2001; Listwan, Van Voorhis \& Ritchey, 2007). Identificou-se também um estudo com adolescentes do sexo feminino no qual o Inventário de Jesness identificou aspectos de personalidade importantes que diferenciaram o grupo de infratoras violentas do grupo de infratoras não violentas (Allen et al., 2003). Contudo, não há estudo, que saibamos, que tenha investigado diferenças entre adolescentes infratores do sexo masculino violentos e não violentos em relação aos atributos de personalidade mensurados pelo Inventário de Jesness.

Sendo assim, o presente estudo teve como objetivo verificar se adolescentes apresentando delinquência persistente, porém diferentes quanto à implicação em delitos caracterizados por violência interpessoal, apresentavam padrões de personalidade diferentes, em termos de traços, crenças, valores e atitudes, de acordo com a avaliação com o Inventário de Jesness.

\section{Método}

A pesquisa aqui relatada tem um delineamento de corte transversal do tipo comparativo, no qual dois grupos de adolescentes em conflito com a lei - violentos e não violentos foram comparados quantitativamente em relação às 13 escalas do Inventário de Jesness. Conforme recomenda a Resolução n. 466/12 do Conselho Nacional de Saúde, o projeto de pesquisa foi encaminhado para análise do Comitê de Ética em Pesquisa com Seres Humanos da faculdade à qual a pesquisa está vinculada, e só teve início mediante aprovação do referido comitê (CAAE 49125415.0.0000.5407).

\section{Participantes}

A amostra desta pesquisa foi constituída por 129 adolescentes do sexo masculino com processos judiciais de execução de medida socioeducativa ativos em um município de médio porte do interior do estado de São Paulo. Os adolescentes tinham, em média, 3,2 (DP $=1,9)$ boletins de ocorrência registrados; idade entre 15 e $19 \operatorname{anos}(\mathrm{M}=17,8 ; \mathrm{DP}=0,8)$; ano escolar com a seguinte distribuição: $5^{\circ}$ ano E. F. (3,9\%), $6^{\circ}$ ano E. F. $\left(6,2^{\%} \%\right), 7^{\circ}$ ano E. F. $(11,6 \%), 8^{\circ}$ ano E. F. $(7,0 \%)$, $9^{\circ}$ ano E. F. (26,4\%), $1^{\circ}$ ano E. M. (31,0\%), $2^{\circ}$ ano E. M. $(10,1 \%)$ e $3^{\circ}$ ano E. M. (3,9\%). A condição socioeconômica, segundo o Critério de Classificação Socioeconômica do Brasil [CCSB] (Abep, 2014), teve a seguinte distribuição: A2 (1,6\%), B1 (1,6\%), B2 $(21,7 \%)$, C1 (43,4\%), C2 (27,1\%) e D $(4,7 \%)$.

\section{Instrumentos}

O Questionário sobre Comportamentos Juvenis (QCJ) acessa informações sobre vários domínios da vida do adolescente. Para o presente estudo, foram considerados apenas os 13 itens que acessam informações sobre as atividades delituosas dos adolescentes. Em cada item, o instrumento requer que o participante responda se alguma vez já apresentou o referido comportamento (no curso de sua vida). Em caso de resposta afirmativa, sucedem questões sobre quantas vezes o participante teve tal 
comportamento nos últimos 12 meses. Entre os 13 delitos investigados, três são considerados violentos por se enquadrarem na definição de violência adotada por este estudo: roubo, lesão corporal e lesão corporal com uso de instrumento. O QCJ foi adequado ao contexto brasileiro por Komatsu (2014) a partir da versão utilizada em Portugal (Castro, Cardoso \& Agra, 2010) e mostrou-se útil em distinguir adolescentes com diferentes níveis de engajamento infracional a partir do autorrelato dos comportamentos delituosos (Komatsu, 2014; Komatsu \& Bazon, 2017).

O Inventário de Jesness - Revisado Brasileiro (IJ-R-Br) avalia crenças, atitudes, percepções, sentimentos e disposições para comportamentos impulsivos e hostis, o que reflete, em boa medida, o modo de funcionamento psicológico do indivíduo em diferentes situações sociais e, em especial, nas que remetem a condutas antissociais. $O$ instrumento tem 160 itens que remetem a afirmações diante das quais $o$ adolescente deve dizer se o conteúdo é "Verdadeiro" ou "Falso". As combinações entre os itens compõem 10 escalas que representam traços de personalidade do indivíduo: Desadaptação Social (DS), Orientação de Valores (OV), Imaturidade (IM), Autismo (AU), Alienação (AL), Agressividade Manifesta (AM), Retraimento-Depressão (RT/DEP), Ansiedade Social (AS), Repressão (REP) e Negação (NEG). Os itens também se relacionam a duas escalas referentes a tipologias descritas na quarta edição do Manual Diagnóstico e Estatístico de Transtornos Mentais (DSM-IV): Transtorno de Conduta (TC) e Transtorno Opositor Desafiador (TOD). Por fim, há também um índice relacionado ao potencial de reincidência de jovens infratores: o Índice de Associabilidade
(IA), calculado por meio da aplicação de uma fórmula que combina escores de oito das escalas de personalidade (DS, OV, AU, AL, AM, RT, AS e $\mathrm{RP}$ ), de modo que o escore produzido possibilita aferir o risco de o comportamento delituoso persistir, em termos de disposição básica e orientação social. As definições e conteúdo das escalas estão amplamente descritos no trabalho de Bazon (2016). O IJ-R ajuda a compreender o adolescente em conflito com a lei, no plano da sua subjetividade e, com isso, a empreender um acompanhamento mais personalizando, o que faz dele um dos instrumentos de avaliação mais utilizados no Sistema de Justiça Juvenil na América do Norte - EUA e Canadá (Semel, 2016).

Adaptado semanticamente para o Brasil, do original (Jesness, 2003), por Manzi-Oliveira (2012), o IJ-R-Br foi testado em suas qualidades psicométricas (Bazon, 2016; Costa, Komatsu \& Bazon, 2017) e teve suas normas por faixa etária também estabelecidas (Bazon, 2016). Nesses estudos, verificou-se que a consistência interna das escalas do IJ-R-Br, aferida por teste-reteste com intervalo de um mês e meio com 38 adolescentes não judicializados, variou de 0,45 a 0,71. Verificouse, ainda, que a consistência interna aferida pelo método das metades com 2.328 adolescentes variou de 0,57 a 0,87. Quanto à validade, as pontuações dos adolescentes infratores/judicializados e não infratores/não judicializados diferiram em 76\% das comparações realizadas para cada escala, por faixa etária de 13 a 18 anos, indicando que as medidas concorrem em um mesmo critério. Além disso, as escalas DS e IA do IJ-R-Br mostraram-se bastante sensíveis em classificar corretamente $79 \%$ e $81 \%$ dos adolescentes infratores/judicializados e $75 \%$ e 
$80 \%$ dos adolescentes não infratores/não judicializados.

\section{Procedimento de coleta}

Os adolescentes foram recrutados em duas Organizações não Governamentais (ONGs) responsáveis pela execução da medida socioeducativa de Liberdade Assistida $(n=45)$ e em uma Unidade de Internação da Fundação Casa (n = 84), todas sediadas no município onde a pesquisa foi realizada, mediante autorização dessas instituições, da autorização dos pais/responsáveis, por meio da assinatura do Termo de Consentimento e aceite dos participantes, manifesto em Termo de Assentimento. Tanto as instituições quanto os participantes e seus responsáveis foram informados quanto ao objetivo, método e aspectos éticos do trabalho, de modo que a coleta de dados dos adolescentes só foi iniciada quando todas as questões relativas à pesquisa estavam esclarecidas.

A coleta de dados se deu de forma individual em uma sala reservada na própria instituição onde os adolescentes foram recrutados. Os instrumentos QJC e IJ-R-Br foram aplicados por um profissional treinado, que leu as perguntas/itens dos instrumentos e assinalou as respostas dadas pelos adolescentes em folhas de respostas específicas. As entrevistas ocorreram entre os meses de janeiro e agosto de 2015.

\section{Procedimento de análise}

Primeiramente, os adolescentes foram divididos em dois grupos, violento $(\mathrm{GV})$ e não violento (GNV), a partir do autorrelato de já terem praticado delito violento ou não, obtido pelo QCJ.
Dessa forma, o GV compreendeu 89 adolescentes com média de idade de 17,9 anos $(\mathrm{DP}=0,7)$ e o GNV compreendeu 40 adolescentes com média de idade de 17,5 anos (DP $=0,9)$. Os grupos não diferiram em relação à escolaridade $\left[\mathrm{X}^{2}(7)=13,7\right.$; $\mathrm{p}$ $=0,06]$ nem à classe socioeconômica $\left[\mathrm{X}^{2}(5)=5,2 ; \mathrm{p}\right.$ $=0,4]$. O GV havia sido detido em média 3,3 vezes, enquanto o GNV havia sido detido em média 2,9, não sendo uma diferença estatisticamente significativa $[\mathrm{t}(115)=1,2 ; \mathrm{p}=0,7]$. Assume-se, portanto, que em termos de escolaridade, classe socioeconômica e número de vezes que já haviam sido apreendidos por cometimento de delitos potenciais variáveis confundidoras - que os grupos se equivaleram.

Por fim, os grupos foram caracterizados, em termos de medidas centrais, em relação aos escores normatizados das 13 escalas do IJ-R-Br e comparados por testes paramétrico ( $\mathrm{t}$ de student) e não paramétrico (U de Mann-Whitney). O uso dos dois testes diminui a chance de falsos positivos, no sentido de que um pode sinalizar uma limitação do outro devido às diferentes distribuições e desvios que o conjunto de variáveis em análise pode assumir (Komatsu, 2017). Como medida de magnitude do efeito, foi calculado o $d$ de Cohen da diferença entre as médias. Devido às múltiplas comparações entre os grupos, o procedimento de Benjamini-Hochberg (Benjamini \& Hochberg, 1995) foi realizado para corrigir o valor $p$ em todas as comparações, resultando em um valor $q$ que foi utilizado como parâmetro para determinar as significâncias estatísticas. 


\section{Resultados}

A Tabela 1 mostra o desempenho dos dois grupos nas 13 escalas do IJ-R-Br e o resultado da comparação dos testes $\mathrm{t}$ de student e U de Mann-
Whitney, que apontaram, igualmente, diferenças significativas em seis escalas (DS, AM, AS, Rep, TC e TOD). Nas outras sete escalas, não foram detectadas diferenças estatisticamente significativas entre os grupos.

Tabela 1. Comparação dos escores de GV e GNV nas escalas do IJ-R-Br

\begin{tabular}{|c|c|c|c|c|c|c|c|c|c|}
\hline & \multicolumn{2}{|c|}{ GNV } & \multicolumn{2}{|l|}{ GV } & \multirow[t]{2}{*}{$d$} & \multicolumn{2}{|c|}{$\begin{array}{c}t \text { de } \\
\text { student }\end{array}$} & \multicolumn{2}{|c|}{$\begin{array}{l}\text { Mann- } \\
\text { Whitney }\end{array}$} \\
\hline & M (DP) & Mdn & M (DP) & Mdn & & $t$ & $p$ & $\bar{U}$ & $p$ \\
\hline DS & $55(12,3)$ & 54,5 & $62,3(10,2)$ & 68 & ,68 & $-3,6$ & ,035* & 1190 & ,038* \\
\hline $\mathrm{OV}$ & $52,3(10,8)$ & 54,5 & $57,2(9,8)$ & 60 & ,48 & $-2,5$ &, 058 & 1299 & ,062 \\
\hline IM & $49,5(12,7)$ & 48 & $54,4(12,7)$ & 55 & ,39 & $-2,1$ & ,077 & 1354 & , 065 \\
\hline $\mathrm{AU}$ & $46,2(12)$ & 43 & $52,7(12,3)$ & 53 &, 53 & $-2,8$ & ,054 & 1235 & ,050 \\
\hline $\mathrm{AL}$ & $55,9(13,9)$ & 55 & $59,1(11,3)$ & 60 & ,26 & $-1,3$ &, 096 & 1534 & 0,10 \\
\hline $\mathrm{AM}$ & $48,3(12,9)$ & 45 & $57,4(12,1)$ & 60 &, 74 & $-3,9$ &, $023^{*}$ & 1065 &, $031 *$ \\
\hline RT/DEP & $49,3(13,2)$ & 50 & $53,9(9,5)$ & 53 &, 43 & -2 & ,085 & 1445 & ,092 \\
\hline AS & $49,8(9,6)$ & 50 & $55(9,7)$ & 57 &, 54 & $-2,9$ &, $046^{*}$ & 1228 &, $042 *$ \\
\hline REP & $59,5(11,3)$ & 60 & $51,6(10,4)$ & 50 &, 74 & 3,9 &, $027 *$ & 1038 &, $019 *$ \\
\hline NEG & $51,1(12,5)$ & 51,5 & $46,3(10,3)$ & 47 & ,43 & 2,1 &, 073 & 1385 & 081 \\
\hline IA & $58,9(12,1)$ & 61 & $63(8,3)$ & 65 & ,43 & $-1,9$ & ,088 & 1364 & ,069 \\
\hline TC & $48,3(13)$ & 50 & $59,7(12,6)$ & 60 & ,89 & $-4,7$ & ,004* & 938 &, $008 *$ \\
\hline TOD & $43,6(9,9)$ & 43 & $51,7(10,3)$ & 53 &, 79 & $-4,2$ & ,015* & 966 &, $012^{*}$ \\
\hline
\end{tabular}

Fonte: Elaborada pelos autores.

\section{Discussão}

O Inventário de Jesness mostrou-se capaz de identificar diferenças importantes entre adolescentes que se envolveram em delitos violentos e adolescentes que não se envolveram. De forma geral, o grupo de adolescentes com prática de delitos violentos obteve pior desempenho, considerando a interpretação teórica do instrumento e de suas escalas. Em relação às seis escalas que diferenciaram os dois grupos, destaca-se a escala de Desadaptação Social (DS), que representa um conjunto de atitudes associadas a necessidades não atendidas, que definem a extensão com a qual o indivíduo partilha padrões adaptativos que demonstram inabilidade em atender, de maneira socialmente aceitável, as demandas do ambiente (Bazon, 2016). Em termos relacionais, indivíduos com pontuações altas nessa escala tendem a apresentar inaptidão nas interações sociais, 
desconfiança e hostilidade em relação ao outro, dificuldades nas funções de controle dos impulsos e comportamentos agressivos (Jesness, 2003; Bazon, 2016). Dessa forma, faz sentido que o GV e o GNV não tenham apenas se diferenciado nessa escala, como também a diferença entre as médias tenha sido grande.

A segunda escala em que os grupos se diferenciaram é a Agressividade Manifesta (AM), que consiste primariamente em itens de natureza afetiva que sugerem a presença de sentimentos de raiva e hostilidade facilmente eliciados (Jesness, 2003). Altas pontuações nessa escala também estão associadas a comportamentos hostis e agressivos, fazendo com que os indivíduos com esse conjunto de atitudes tendam a ser mais reativos $\mathrm{e}$ a cometerem delitos violentos, o que também explica o fato de o GV ter pontuação bem mais elevada que o GNV (Jesness, 2003; Bazon, 2016).

Em relação à escala de Ansiedade Social (AS), que remete a sentimentos de ansiedade e desconforto emocional nas interações sociais/ relações interpessoais, o $G \mathrm{~V}$ novamente pontuou mais alto que o GNV. Jesness (2003) aponta que não há diferenças importantes entre infratores e não infratores nesses atributos, todavia, Costa, Komatsu e Bazon (2017), em estudo com adolescentes brasileiros, verificaram que infratores/judicializados pontuaram mais alto que os não infratores/não judicializados, a partir da segunda metade da adolescência. Sendo essa escala composta por itens relacionados principalmente a situações ansiogênicas na interação com o outro, pode-se supor que essa ansiedade é causada por deficit de repertório social, que por sua vez está relacionado a uma probabilidade maior de se recorrer a métodos/estratégias para atender necessidades ou para solucionar problemas pessoais que não são socialmente aceitáveis, o que pode incluir o uso da violência (OMS, 2009). Observa-se ainda a proximidade dessa escala com o conceito de Afetividade Negativa, que remete à tendência em experienciar estados afetivos negativos como raiva, ansiedade e estresse. Estudos indicam forte relação entre esses traços disposicionais e comportamentos violentos (Capaldi, Knoble, Shortt \& Kim, 2012; Moffitt, Krueger, Caspi \& Fagan, 2000).

Outra escala que também diferenciou os grupos foi a de Repressão (Rep), a única em que o GNV pontuou mais alto que o GV. Os itens dessa escala apontam para características de personalidade de "hipernormalidade", como se tudo estivesse sempre bem, o que refletiria a tendência em reprimir da consciência qualquer sentimento ou emoção negativa, como raiva e desgosto (Jesness, 2003; Bazon, 2016). No geral, adolescentes infratores pontuam mais alto que adolescentes não infratores (Costa, Komatsu \& Bazon, 2017; Jesness, 2003). A questão aqui é, todavia, o que explica o fato de infratores não violentos pontuarem mais alto que infratores violentos. Pode-se hipotetizar que esses últimos lidam com realidades mais difíceis de serem reprimidas, como, talvez, situações concretas de violência, o que os tornariam menos propensos a ignorar os fatos difíceis da vida que levam. Adicionalmente, pode-se pensar que infratores violentos apresentam mais carência de recursos para lidar com sentimentos ruins ou difíceis que os não violentos, tendendo, portanto, a agir em função desses. Nessa linha de pensamento, vale ressaltar o fato de essa escala mostrar-se negativamente correlacionada com a escala Psychopathic Deviate, que mensura dimensões de conflito, briga, raiva e desrespeito pelas regras da 
sociedade, do Minnesota Multiphasic Personality Inventory (Jesness, 2003).

As últimas duas escalas que diferenciaram os grupos foram a de Transtorno de Conduta (TC) e a de Transtorno Opositivo Desafiador (TOD), ambas com itens que remetem aos critérios para diagnóstico dos transtornos homônimos, descritos no DSM-IV (Jesness, 2003). Entre todas as escalas, a TC foi a que apresentou maior diferença entre as médias dos grupos, o que era esperado, uma vez que seus itens remetem diretamente a condutas violentas, por exemplo: "Eu já fui mal com pessoas ou animais" e "Eu já usei uma arma que poderia ter machucado alguém". Dessa forma, o resultado obtido com essa escala reforça a validade concorrente do instrumento, mas não contribui para explicar o porquê de os grupos diferirem. Já a escala de TOD apresenta itens que remetem a condutas de enfrentamento, mas também a conteúdos atitudinais relacionados a situações de desobediência a figuras de autoridade. Sendo os delitos violentos uma modalidade inserida em uma grande classe de comportamentos que ferem normas e desafiam as autoridades (que são responsáveis por regular/controlar esse tipo de conduta), pode-se supor uma correlação positiva entre comportamentos violentos e valores, atitudes e ações de desobediência, o que explicaria o fato do GV ter uma pontuação maior que GNV também nessa escala. Ademais, na linha do proposto por Aebi et al. (2015), a partir do estudo que realizaram com adolescentes infratores, o Transtorno em questão (TOD), sendo multidimensional, compreenderia uma dimensão de Irritabilidade que se mostrou fortemente associada à reincidência em delitos violentos.

$$
\text { Passando para as escalas que não }
$$
diferenciaram os grupos, sete mostraram-se indiferentes quanto ao fator da violência: Orientação de Valores, Imaturidade, Autismo, Alienação, Retraimento/Depressão, Negação e o Índice de Associabilidade. Orientação de Valores (OV) é composta por itens relacionados a crenças e atitudes favoráveis a (ou que naturalizam) comportamentos antissociais, como "A maioria das pessoas engana um pouco para conseguir dinheiro”, “A maioria dos policiais não é inteligente" e "Não parece ser errado roubar de comerciantes desonestos", por isso ela apresenta boa acurácia em discriminar adolescentes infratores de não infratores (Costa, Komatsu \& Bazon, 2017). Outros itens dessa escala referem-se à presença de inquietações, ansiedade, pensamentos estranhos e alienação social. No entanto, seus itens não remetem a conteúdos relacionados à violência, o que pode explicar o porquê de ela não diferenciar, em meio aos adolescentes infratores, aqueles que fazem uso da violência daqueles que não fazem.

A Imaturidade (IM) reflete a extensão com a qual o indivíduo falha em demonstrar respostas, atitudes e percepções que são esperadas por alguém de sua idade (Jesness, 2003). Pontuar baixo em IM indica uma orientação realista maior; escores muito baixos, porém, sugerem pessimismo e cinismo acentuados (Bazon, 2016). Adolescentes infratores tendem a pontuar mais alto nessa escala (Jesness, 2003), o que foi constatado no contexto brasileiro, no qual adolescentes infratores/judicializados tiveram maior pontuação que os não infratores/não judicializados (Costa, Komatsu \& Bazon, 2017). Em termos de reabilitação, Cowden, Peterson e Pacht (1969) encontraram que adolescentes infratores que pontuam mais alto têm piores prognósticos. No 
entanto, a escala não apresentou sensibilidade para a questão da violência, provavelmente pelo conteúdo de seus itens referirem-se a vontades, percepções e situações cotidianas que também não estão relacionadas à conduta violenta.

O Autismo (AU) é definido como a tendência de o pensamento do indivíduo ser regulado excessivamente por necessidades pessoais, de modo que a percepção de si e do outro e o planejamento da ação apresentam-se irrealistas (Jesness, 2003). Nesse sentido, indivíduos que pontuam alto costumam atribuir uma importância exagerada às próprias necessidades, formando uma imagem amplificada de si próprio, juntamente com a experimentação de uma dificuldade para diferenciar o que é seu do que não é (Bazon, 2016). Os estudos de validade do Inventário apontam que indivíduos que pontuam alto nessa escala tendem a apresentar comportamentos hostis e agressivos (Jesness, 2003), por isso era esperado que o GV apresentasse uma média maior que o GNV, o que não aconteceu. Uma possível explicação para isso é o fato de o teor dos itens que remetem a conteúdos agressivos referirem-se mais propriamente a crenças e atitudes gerais, como expressam os itens "Para o meu tamanho, eu sou um cara durão" e "Às vezes, o único meio de acertar um negócio é usando a força". Essas crenças/atitudes, embora possam ter certo conteúdo hostil ou agressivo, não parecem ser suficientes para sustentar a implicação em delitos violentos, sendo, portanto, indiferentes para discriminar os grupos.

$$
\text { A escala de Alienação (Al) refere-se à }
$$
desconfiança e ao estranhamento em relação ao outro, de modo que indivíduos que pontuam alto nessa escala costumam ser céticas e críticas em relação às pessoas, repercutindo em uma atitude predominantemente hostil em suas respostas (Jesness, 2003). Embora a descrição da escala possa sugerir que o $\mathrm{GV}$ devesse pontuar mais alto que GNV, a hostilidade presente nas atitudes de quem pontua alto nessa escala não está diretamente ligada à conduta violenta, que fere ou apresenta risco de ferir a integridade física do outro, mas sim a uma hostilidade velada, de afastamento em relação ao outro, representada em itens como: "É muito melhor não ter confiança em ninguém", "Pouco me importa se as pessoas gostam ou não de mim", "Nunca se pode acreditar no que os pais e mães dizem". Desse modo, embora os estudos de validade indiquem que adolescentes infratores pontuam mais que adolescentes não infratores (Costa, Komatsu \& Bazon, 2017; Jesness, 2003), essa escala não diferencia infratores violentos de não violentos.

A escala de Retraimento/Depressão (RT/DEP) indica o quanto o indivíduo tende a resolver seus problemas de falta de satisfação consigo mesmo e com os outros por meio de fuga passiva ou isolamento (Jesness, 2003). Pessoas com alta pontuação costumam se perceber como deprimidas, insatisfeitas consigo mesmas e não compreendidas, preferindo ficar sozinhas, mesmo que acompanhadas por um sentimento de solidão (Jesness, 2003). Os itens em RT/DEP não remetem a conteúdos violentos, mas sim a uma tendência a deprimir e isolar-se, e em mostrar desinteresse por atividades cotidianas, como na afirmativa " $\mathrm{Na}$ minha vida nunca aconteceu uma grande coisa". Assim, a pontuação obtida nessa escala não é preditiva de delinquência, mas pode indicar características de funcionamento do indivíduo que precarizam ainda mais as possibilidades de experimentar interações sociais positivas (Bazon, 
2016), por isso também era esperado que os grupos não se diferenciassem nessa dimensão.

A última escala é a Negação (NEG), cujos itens estão relacionados a problemas familiares, inadequações pessoais e infelicidade. A escala avalia a tendência da pessoa a, embora percebendo/apreendendo os problemas na própria família e em si mesmo, não pensar nisso. Uma pontuação relativamente elevada nessa escala é indicativa de ajustamento emocional positivo, denotando certo otimismo (Jesness, 2003) e presença de recursos para lidar com sentimentos ruins. No transcorrer da adolescência, conforme vão adquirindo mais capacidade de perceber os problemas da própria família, adolescentes infratores tendem a pontuar mais baixo que adolescentes não infratores nessa escala (Costa, Komatsu \& Bazon, 2017). Nesse sentido, esperavase que os dois grupos pontuassem abaixo da média, mas não necessariamente que fossem diferentes entre si.

Por fim, os grupos também não se diferenciaram quanto ao Índice de Associabilidade (IA), composto por uma combinação de itens das outras escalas com a função de distinguir adolescentes infratores e não infratores com maior acurácia, em razão de sua orientação social (Jesness, 2003). Dessa forma, o IA sintetiza informações que indicam uma tendência para comportar-se de um modo a transgredir regras sociais estabelecidas, podendo ser útil na avaliação do potencial de risco de reincidência infracional (Bazon, 2016). Como os dois grupos aqui comparados são compostos por adolescentes infratores que apresentam uma delinquência persistente, não era esperado que se distinguissem quanto à pontuação no IA.
Em suma, as escalas que diferenciaram os grupos são aquelas relacionadas a características de personalidade que remetem a problemas de controle dos impulsos e manejo da raiva, e de maior hostilidade e atitudes agressivas. Esses atributos remetem a traços, valores e atitudes que favorecem o uso da violência como recurso para alcançar objetivos ou solucionar problemas (violência utilitária) ou, ainda, tornam o indivíduo mais propício a reagir de forma agressiva diante de estímulos suscetíveis de eliciar respostas emocionais (violência reativa). Em contrapartida, as escalas que não diferenciaram podem ser alocadas em dois grupos: as que avaliam uma tendência a transgredir regras sociais e cometer delitos, de forma geral, sem estarem relacionadas à violência, e aquelas relacionadas a aspectos específicos do funcionamento psicológico, como a escala de Retraimento/Depressão, que não se relaciona com comportamentos antissociais, de forma gral, tampouco com comportamentos violentos de forma específica.

Uma observação importante a se fazer é que essas características de personalidade, e por conseguinte, os padrões de comportamentos violentos, são desenvolvidos no curso da vida, nas interações sociais, nos mais diversos contextos de vida, de modo que programas de prevenção da violência podem ser pensados no sentido de promover, em crianças e adolescentes, habilidades de coping como controle (manejo adequado) de sentimentos negativos e estratégias para solução de problemas, no escopo da Política Nacional de Promoção da Saúde (PNPS, 2006). Nessa esteira, pode-se pensar em diferentes ações eficientes, entre as quais se destacam, conforme revisão apresentada por Piquero, Jennings e Reingle (2017), aquelas 
relacionadas a treino de habilidades parentais e psicoeducação, por meio de visitas domiciliares (que, no Brasil, poderiam ser incorporadas na Estratégia Saúde da Família), de modo que os cuidadores aprendam a discriminar e a reduzir suas práticas negativas (e os efeitos destas nas crianças e nos adolescentes), bem como a atuarem de forma mais positiva.

Em se tratando de adolescentes infratores/judicializados, em acompanhamento socioeducativo, o atendimento psicossocial a eles dispensado, no escopo da atenção integral a sua saúde, deve vir ao encontro de suas necessidades desenvolvimentais, conforme a avaliação que se fizer de cada um, de forma que o acompanhamento seja personalizado. Assim, os aspectos psicossociais que podem ter contribuído para que se envolvessem em atos infracionais e que riscam favorecer a persistência do comportamento delituoso/infracional devem ser foco do acompanhamento, assim como aqueles suscetíveis de colocar o jovem numa trajetória de implicação em delitos violentos merecem atenção especial, incluindo-se aí as dimensões da personalidade destacadas no presente estudo, até porque elas são maleáveis conforme o referencial a partir do qual foram concebidas (Jesness, 2003; Bazon, 2016). Nessa óptica, há que se salientar que as ações de promoção de saúde mental e/ou de prevenção e de cuidado de transtornos mentais, em adolescentes infratores em privação liberdade, como parte das ações de promoção, proteção e recuperação da saúde dessa população, requerem um ambiente de pleno respeito aos direitos humanos e à integridade física e mental dos adolescentes, conforme reza a Política Nacional de Atenção Integral à Saúde de Adolescentes em Conflito com a Lei em Regime de
Internação e Internação Provisória (Portaria n. 1.082, 2014), do contrário, a própria execução desse tipo de medida judicial é suscetível de funcionar como fator de risco à saúde metal dos adolescentes, aumentando, assim, sua vulnerabilidade à delinquência violenta.

\section{Considerações finais}

A violência de natureza interpessoal tem um grande e nefasto impacto na vida em sociedade. No Brasil, os jovens constituem um dos segmentos mais afetados, seja como vítima, seja como perpetrador. No que respeita ao segmento específico dos adolescentes em conflito com a lei, entender porque alguns apresentam uma delinquência persistente e, com isso, se implicam em delitos violentos, é fundamental à proposição de ações adequadas, que concorram à alteração das trajetórias comportamentais, em contraposição à mera reatividade negativa, que conclama por mais dura repressão/punição a eles, em vista ao direito que eles têm de receber os serviços adequados à promoção e à proteção ao pleno desenvolvimento humano.

O estudo aqui relatado buscou oferecer uma colaboração nessa direção, focalizando aspectos de personalidade que regulam um funcionamento psicológico que parece associado a essa conduta. Denotou-se que os adolescentes infratores investigados, que têm histórico de implicação em violência, apresentaram mais marcadamente características de personalidade que remetem a problemas de controle dos impulsos e manejo da raiva, e de maior hostilidade e atitudes agressivas. À luz do referencial teórico adotado, que permitiu tal avaliação, essas características são 
maleáveis - portanto suscetíveis a intervenções psicossociais - e seriam construídas no curso da vida, por intermédio das interações, nos múltiplos contextos de vida, o que pode, inclusive, incluir a experiência de institucionalização, em que certas práticas podem reforçar essas características.

Em termos instrumentais, tem-se que o IJ$\mathrm{R}-\mathrm{Br}$ se mostrou capaz de diferenciar adolescentes infratores violentos e não violentos - como grupos -, revelando as características de funcionamento psicológico relevantes ao objetivo da pesquisa. Isso leva a crer que o IJ-R-Br pode vir a ser uma ferramenta útil aos profissionais da saúde, no âmbito da Justiça Juvenil, capaz de auxiliar na elaboração dos Projetos Terapêuticos Singulares, que podem ser integrados aos Planos Individuais de Atendimento (PIA) previstos no Sistema Nacional de Atendimento Socioeducativo (Lei n. 12.594, 2012), de modo a atender às complexas necessidades dessa população. É certo que tal auxílio, no âmbito das avaliações individuais, deve ser concebido como complementar; assim como se recomenda que em toda avaliação psicológica as pontuações em instrumentos padronizados devem, sempre, ser consideradas conjuntamente a outras informações, e interpretadas em uma perspectiva mais ampla, em relação ao contexto de vida dos indivíduos (Werlang, Villemor-Amaral \& Nascimento, 2010).

Por fim, é importante destacar a importância de novas investigações que possam corroborar os achados, e permitam generalizá-los, assim como ultrapassar os limites metodológicos do presente estudo. Um primeiro ponto seria a replicação do estudo em distintas amostras de adolescentes infratores, em distintas regiões do país, buscando controlar a variável institucionalização.
Igualmente importante seria a replicação do estudo em amostras de adolescentes da população geral, afora a característica de judicialização que caracterizou a aqui estudada. Por último, com vistas a investigar as efetivas relações de determinação entre os aspectos de personalidade destacados e os padrões de comportamento violento, seria necessária a realização de estudos longitudinais, com foco no desenvolvimento do comportamento antissocial e nas variáveis pessoais e sociais a ele associadas, com a coleta de dados em múltiplos pontos do tempo, empregando múltiplas estratégias (outras além da aplicação de instrumentos padronizados, de autorrelato, com o próprio adolescente).

\section{Referências}

Abep. Associação Brasileira de Empresas de Pesquisa. (2014). Critérios de classificação econômica 2014. Recuperado em 25 dezembro, 2017, de http://abep.org/Servicos/Download.aspx?id $=01$

Achenbach, T. M., \& Rescorla, L. A. (2006). Developmental Issues in Assessment, Taxonomy, and Diagnosis of Psychopathology: Life Span and Multicultural Perspectives. In D. Cicchett \& D. Cohen (Orgs.). Developmental Psychopatology: Theory and Method (Vol. 1, 2th ed.), Hoboken, New Jersey: John Wiley \& Sons Inc.

Aebi, M., Barra, S., Bessler, C., Steinhausen, H., Walitza, S., \& Plattner, B. (2015). Oppositional Defiant Disorder Dimensions and Subtypes among Detained Male 
Adolescent Offenders. Journal of Child

Psychology and Psychiatry, 57(6), 729-736.

Allen, J., Rupert, V., Spatafora, K., Windell, J. O., Gaulier, B., \& Conti, K. L. (2003).

Differentiating Violent from Nonviolent

Female Offenders Using the Jesness

Inventory. Personality and Individual Differences, 35(1), 101-108.

Andrews, D. A., \& Bonta, J. (2010). The Psychology of

Criminal Conduct. New Providence:

LexisNexis - trademark of Reed Elsevier.

Bazon, M. R. (2016). Avaliação psicológica de adolescentes em conflito com a lei: validaşão do Inventário de Jesness - Revisado. Tese de livre docência, Faculdade de Filosofia, Ciências e Letras de Ribeirão Preto, Universidade de São Paulo, Ribeirão Preto, São Paulo.

Benda, B. B., Corwyn, R. F., Toombs, N. J. (2001). Recidivism among Adolescents Serious Offenders: Prediction of Entry into the Correctional System for Adults. Criminal Justice and Behavior, 28(5), 588-613.

Benjamini, Y., \& Hochberg, Y. (1995). Controlling the False Discovery Rate: A Practical and Powerful Approach to Multiple Testing. Journal of the Royal Statistical Society Series B: Statistical Methodology, 57(1), 289-300.

Capaldi, D. M., Knoble, N. B., Shortt, J. W., \& Kim, H. K. (2012). A Systematic Review of Risk Factors for Intimate Partner Violence. Partner Abuse, 3(2), 231-280.

Castro, J., Cardoso, C., \& Agra, C. (2010). Projecto: Observatório da Delinquência Juvenil.
Relatório Final. Escola de Criminologia, Faculdade de Direito da Universidade do Porto. (Não publicado).

Cerqueira, D., Lima, R. S., Bueno, S., Valencia, L. I., Hanashiro, O., Machado, P. H. G., \& Lima, A. S. (2017). Atlas da violência 2017. Recuperado em 25 dezembro, 2017, de http://www.ipea.gov.br/portal/images/1706 02_atlas_da_violencia_2017.pdf.

Chernyshev, B. V., Lazarev, I. E., \& Chernysheva, E. G. (2013). Temperament: An EventRelated Potential Study Using the Oddball Paradigm. Psychology \& Neuroscience, 6(3), 235245.

Cook, E. C., Pflieger, J. C., Connell, A. M., \& Connell, C. M. (2015). Do Specific Transitional Patterns of Antisocial Behavior During Adolescence Increase Risk for Problems in Young Adulthood?. Journal of Abnormal Child Psychology, 43(1), 95-106.

Cowden, J. E., Peterson, W. M., \& Pacht, A. R. (1969). The MCI vs. The Jesness Inventory as a Screening and Classification Instrument at a Juvenile Correctional Institution. Journal of Clinical Psychology, 25(1), 57-60.

Costa, R., Komatsu, A. V., \& Bazon, M. R. (2017). Psychological Assessment of Adolescent Offenders. International Annals of Criminology, 55(1), 60-77.

Datafolha. (2013). Pesquisa Nacional de Vitimização. Recuperado em 25 dezembro, 2017, de http:/ /www.crisp.ufmg.br/wpcontent/uploads/2013/10/Sumario_SENAS P_final.pdf. 
Dias, J., Dias, A. R. C., Formiga, N. S., \& Gonçalves, R. (2016). Juvenile Delinquency and Victimization in Cape Verde: Indicators of Prevalence and Characterization. EurekaRevista Científica de Psicologia, 13(1), 24-38.

Díaz-Galván, K. X., Ostrosky-Shejet, F., \& Romero-Rebollar, C. (2015). Cognitive and Affective Empathy: The Role in Violent Behavior and Psychopathy. Revista Médica del Hospital General de México, 78(1), 27-35.

Dodge, K. A. (2011). Social Information Processing Patterns as Mediators of the Interaction between Genetic Factors and Life Experiences in the Development of Aggressive Behavior. In P. R. Shaver \& M. Mikulincer (Eds.). Human Aggression and Violence: Causes, Manifestations, and Consequenses. Washington, DC: American Psychological Association.

Fórum Brasileiro de Segurança Pública. (2013). Anuário de Segurança Pública. Recuperado em 25 dezembro, 2017, de http://forumseguranca.org.br/storage/7_an uario_2013-corrigido.pdf.

Fórum Brasileiro de Segurança Pública. (2015). Anuário de Segurança Pública. Recuperado em 25 dezembro, 2017, de http://www.forumseguranca.org.br/atividad es/anuario/.

Fórum Brasileiro de Segurança Pública. (2016a). Atlas da Violência. Recuperado em 25 dezembro, 2017, de http://ipea.gov.br/portal/images/stories/P
DFs/nota_tecnica/160322_nt_17_atlas_da_ violencia_2016_finalizado.pdf.

Fórum Brasileiro de Segurança Pública. (2016b). Anuário de Segurança Pública. Recuperado em 25 dezembro, 2017, de http://www.forumseguranca.org.br/storage/ 10_anuario_site_18-11-2016-retificado.pdf.

Gendreau, P., Little, T., \& Goggin, C. (1996). A Meta-Analysis of the Predictors of Adult Offender Recidivism: What Works!. Criminology, 34, 575-607.

Howard, R. (2015). Personality Disorders and Violence: What is the Link? Borderline Personality Disorder and Emotion Dysregulation, 2, 12.

Jesness, C. F. (2003). Jesness Inventory-Revised. Technical Manual. North Tonawanda, USA: MHS.

Komatsu, A. V. (2014). Comportamentos antissociais em adolescentes do sexo masculino: estudo exploratório na cidade de Ribeirão Preto - SP. Dissertação de mestrado, Faculdade de Filosofia, Ciências e Letras de Ribeirão Preto, Universidade de São Paulo, Ribeirão Preto, São Paulo.

Komatsu, A. V. (2017). Comparação dos poderes dos testes t de Student e Man-Whitney Wilcoxon pelo método de Monte Carlo. Revista da Estatistica UFOP, 6, 121-127.

Komatsu, A. V., \& Bazon, M. R. (2015). Caracterização de adolescentes do sexo masculino em relação a comportamentos antissociais. Revista Latinoamericana de Ciencias Sociales, Ninezy Juventud, 13(2), 725-735. 
Komatsu, A. V., \& Bazon, M. R. (2017). Personal Differences among Brazilian Adolescents with Different Levels of Criminal Engagement. International Journal of Criminology \&. Sociology, 6, 65-74.

Komatsu, A. V., \& Bazon, M. R. (2018). Fatores de risco e de proteção para emitir delitos violentos: revisão sistemática da literatura. Perspectivas em Psicologia, 22(1), 180-206.

Le Blanc, M. (2003). Trajetórias de delinquência comum, transitória e persistente: uma estratégia de prevenção diferencial. In I. Alberto (Org.). Comportamento antissocial: escola e familia (pp. 31-80). Coimbra: Centro de Psicopedagogia da Universidade de Coimbra.

Larsen, R. J., \& Buss, D. M. (2009). Personality Psychology: Domains of Knowledge about Human Nature. New York: McGraw-Hill Education.

Lei n. 12.594, de 18 de janeiro de 2012. (2012). Institui o Sistema Nacional de Atendimento Socioeducativo (Sinase), regulamenta a execução das medidas socioeducativas destinadas a adolescente que pratique ato infracional; e altera as Leis $\mathrm{n}^{\circ} \mathrm{s} 8.069$, de 13 de julho de 1990 (Estatuto da Criança e do Adolescente); 7.560, de 19 de dezembro de 1986, 7.998, de 11 de janeiro de 1990, 5.537, de 21 de novembro de 1968, 8.315, de 23 de dezembro de 1991, 8.706, de 14 de setembro de 1993, os Decretos-Leis nos 4.048, de 22 de janeiro de 1942, 8.621, de 10 de janeiro de 1946, e a Consolidação das Leis do Trabalho (CLT), aprovada pelo Decreto-Lei n ${ }^{\circ} 5.452$, de $1^{\circ}$ de maio de 1943. Brasília, DF. Recuperado em 25, dezembro, 2017, de
http://www.planalto.gov.br/ccivil_03/_Ato20112014/2012/Lei/L12594.htm.

Listwan, S. J., Van Voorhis, P., \& Ritchey, P. N. (2007). Personality, Criminal Behavior, and Risk Assessment: Implications for Theory and Practice. Criminal Justice and Behavior, 34(1).

Loeber, R., Farrington, D. P., \& Petechuk, D. (2013). Bulletin 1: From Juvenile Delinquency to Young Adult Offending (Study Group on the Transitions between Juvenile Delinquency and Adult Crime). Report of U.S. Department of Justice. Retrieved December 25, 2017, from https://www.ncjrs.gov/pdffiles1/nij/grants/ 242931.pdf.

Manzi-Oliveira, A. B. (2012). Avaliação de adolescentes em conflito com a lei: adaptação transcultural do Inventário de Personalidade de Jesness. Dissertação de mestrado, Faculdade de Filosofia, Ciências e Letras de Ribeirão Preto, Universidade de São Paulo, Ribeirão Preto, São Paulo.

Marshall, M. A., \& Brown, J. D. (2006). Trait Aggressiveness and Situational Provocation: A Test of the Traits as Situational Sensitivities (TASS) Model. Personality and Social Psychology Bulletin, 32(8).

McGee, T. R., \& Farrington, D. P. (2015).

Developmental and Life-Course Theories of Crime. In A. Piquero (Org.). The Handbook of Criminological Theory (336-354).

McGuire, J. (2009). Reducing Personal Violence: Risk Factors and Effective Interventions. In 
S. Hodgins, E. Viding \& A. Plodowski (Eds.). The Neurobiological Basis of Violence: Science and Rehabilitation (pp. 287-327). New York: Oxford University Press.

Moffitt, T. E. (1993). Adolescence-Limited and Life-Course Persistent Antisocial Behavior: A Developmental Taxonomy. Psychological Review, 100, 674-701.

Moffitt, T. E., Krueger, R. F., Caspi, A., \& Fagan, J. (2000). Partner Abuse and General Crime: How Are They the Same? How Are They Different?. Criminology, 38, 199-232.

Organização Mundial de Saúde - OMS. (2002). Relatório mundial sobre violência e saúde. Genebra: WHO. Recuperado em 25 dezembro, 2017, de https://www.opas.org.br/wpcontent/uploads/2015/09/relatoriomundial-violencia-saude.pdf.

Organização Mundial de Saúde - OMS. (2009). Preventing Violence by Developing Life Skills in Children and Adolescents. Series of Briefings on Violence Prevention. Malta: WHO press. Retrieved December 25, 2017, from

http://apps.who.int/iris/bitstream/10665/4 4089/1/9789241597838_eng.pdf.

Pickles, A., \& Hill, J. (2006). Developmental Pathways. In D. Cicchett \& D. Cohen (Orgs.). Developmental Psychopatology: Theory and Method (Vol. 1, 2th ed.) Hoboken, New Jersey: John Wiley \& Sons Inc.
Pinsoneault, T. B. (2006). Updating the Jesness Inventory Randomness Validity Scales for the Jesness Inventory - Revised. Journal of Personality Assessment, 86(2), 190-195.

Piquero, A., Jennings, W. G., \& Reingle, J. M. (2017). Life-Course Development and Crime: An Introduction. In Preventing Crime and Violence (Vol. 2, pp. 91-99). New York, NY: Springer International Publishing.

Portaria n. 399, de 22 de fevereiro de 2006. (2006).

Divulga o Pacto pela Saúde 2006 Consolidação do SUS e aprova as Diretrizes Operacionais do Referido Pacto. Brasília, DF. Recuperado em 25 dezembro, 2017, de https://bvsms.saude.gov.br/bvs/saudelegis/ gm/2006/prt0399_22_02_2006.html.

Portaria n. 1.082, de 23 de maio de 2014. (2014).

Redefine as diretrizes da Política Nacional de Atenção Integral à Saúde de Adolescentes em Conflito com a Lei, em Regime de Internação e Internação Provisória (PNAISARI), incluindo-se o cumprimento de medida socioeducativa em meio aberto e fechado; e estabelece novos critérios e fluxos para adesão e operacionalização da atenção integral à saúde de adolescentes em situação de privação de liberdade, em unidades de internação, de internação provisória e de semiliberdade. Brasília, DF, 2014.

Recuperado em 25 dezembro, 2017, de http://bvsms.saude.gov.br/bvs/saudelegis/g m/2014/prt1082_23_05_2014.html.

Semel, R. A. (2016). Incorporating the Jesness Inventory-Revised (JI-R) in a Best-Practice 
Model of Juvenile Delinquency Assessments.

Journal of Forensic Psychology Practice, 16.

Stoddard, S. A., Varela, J. J., \& Zimmerman, M. A.

(2015). Future Expectations, Attitude

Towards Violence, and Bullying Perpetration

During Early Adolescence: A Mediation

Evaluation. Nursing Research, 64(6), 422-433.

Tedeschi, J. T., \& Felson, R. B. (1994). Violence,

Aggression, and Coercitive Actions. Washington:

American Psychological Association.

Waiselfisz, J. J. (2014). Mapa da Violência 2014: os jovens do Brasil. Rio de Janeiro: Cebela-

Flasco.

Werland, B. S. G., Villemor-Amaral, A. E., \& Nascimento, R. S. G. F. (2010). Avaliação psicológica, testes e possibilidades de uso. In Avaliação psicológica: diretrizes na regulamentação da profissão (1 a ed.). Brasília: Conselho Federal de Psicologia.

Winter, K., Spengler, S., Bermpohl, F., Singer, T., \& Kanske, P. (2017). Social Cognition in Aggressive Offenders: Impaired Empathy, But Intact Theory of Mind. Scientific Reports, 7, 670. 